\title{
Construire sur les dix années passées, les dix ans à venir : apprendre de la communication dans le cas des politiques publiques favorables à la santé au Québec, Canada
}

\author{
> Pernelle Smits ${ }^{a}{ }^{*}$, Johanne Préval $^{b}$ et Jean-Louis Denis ${ }^{c}$ \\ a Professeure Université de Laval, Chercheure associée à l'ENAP, \\ 4750 Henri Julien, H2T 3 E5 Montréal. Canada \\ ' Professionnelle de recherche à L'ENAP, 4750 Henri Julien, H2T 3 E5 Montréal. Canada \\ cProfesseur titulaire à L'ENAP, 4750 Henri Julien, H2T 3E5 Montréal. Canada
}

\section{Résumé}

L'article vise à apporter une réflexion sur les apprentissages issus de plusieurs années d'implantation d'une politique publique transversale touchant l'ensemble des ministères, ici les politiques publiques favorables à la santé (PPFS). À partir d'une analyse des menaces passées, actuelles et à court terme pour la mise en œuvre des PPFS au Québec, nous dégageons des pistes d'action pour le futur. Les menaces ont été identifiées à partir d'entrevues avec les acteurs œuvrant à la prise en compte de la santé dans les politiques publiques. Notre analyse est centrée sur les menaces relatives aux aspects communicationnels.

La démarche d'identification des pistes d'action procède en quatre étapes : la documentation des menaces, l'identification d'options répondant aux menaces, la documentation de résultats probants, la possibilité d'implantation dans le contexte local. Cette démarche nous paraît être une alternative relativement rapide et systématique pour documenter une situation et en déduire logiquement des axes d'intervention. Centrer l'analyse sur les aspects communicationnels apparaît pertinent pour approfondir les menaces et dégager des pistes d'action spécifiques aux politiques transversales. D'autres initiatives transversales, comme les stratégies de développement durable, de lutte contre la pauvreté, de lutte contre le changement clinique, pourraient bénéficier d'une telle démarche.

(c) 2015 IDMP/Lavoisier SAS. Tous droits réservés

Mots clés : politiques publiques favorables à la santé, analyse des menaces, action horizontale, évaluation d'impacts en santé, communication.

*Auteur correspondant : pernelle.smits@enap.ca

doi : 10.3166/pmp.31.79-98 @ 2015 IDMP/Lavoisier SAS. Tous droits réservés 


\section{Abstract}

Planning the next ten years upon the past ten: learning from communication in the case of Health in Public Policies in Quebec, Canada. This article intends to draw lessons after years of implementation of whole of government public policies where most ministries take action. We study the case of health in public policies as implemented for more than 10 years in Quebec, Canada. First we describe the different waves of implementation, then current threats are extracted. To draw practical lessons, we borrow from a step-wise analysis that addresses first the threats related to communication, propose solutions from the literature, document proven practical solutions, and then deduct actions that are relevant in the current context. Data are mainly collected from reports on the implementation periods and from semi-structured interviews with managers and professionals in charge of implementing health in public policies in Quebec. The analytic procedure appears as a relatively fast and straightforward way to propose future steps in the implementation of complex whole of government initiatives. Sustainable development strategy, strategies to fight against poverty and other horizontal public actions might benefit from this analytic procedure.

๑ 2015 IDMP/Lavoisier SAS. Tous droits réservés

Keywords: whole of government, threats analysis, health in all policies, health impact assessment, communication.

\section{Introduction}

Depuis une décennie, l'Organisation Mondiale de la Santé a lancé l'initiative évaluations d'impacts en santé (EIS). Plusieurs pays se sont lancés dans les EIS ou des approches semblables (CRICH, 2011) telles que santé dans toutes les politiques publiques, ou politiques publiques favorables à la santé (PPFS) (Buckett, 2010; Kickbusch et Buckett, 2010). Le Bureau européen de l'Organisation Mondiale de la Santé, par le Consensus de Göteborg, définit l'EIS comme une « combinaison de procédures, méthodes et outils qui permettent de juger les effets possibles d'une politique, d'un programme ou d'un projet sur la santé de la population et la distribution de ces effets au sein de la population » (WHO, 1999). L'EIS permet d'éclairer la prise de décision au regard des impacts de futurs projets sur la santé et de proposer des moyens pour pallier les effets négatifs anticipés, et optimiser les effets positifs anticipés (St-Pierre, Vienst et Tremblay, 2012). L'approche des PPFS s'inscrit dans une logique similaire à celle des EIS en faisant de la santé une préoccupation pour plusieurs secteurs de l'administration publique.

Parmi l'ensemble de ces approches, les administrations publiques ont fait des choix différents : multiplier des projets d'EIS, impliquer directement le Ministère de la Santé, institutionnaliser la procédure par une loi et des appuis réglementaires, élargir les EIS aux PPFS. Entre 2000 et 2007, 180 articles sur les EIS ont été publiés dans des revues scientifiques et on compte une quarantaine de guides sur les EIS (Valentini et SaintPierre, 2012). En Europe et en Océanie, diverses pratiques d'EIS ont été répertoriées particulièrement dans les secteurs du transport, du logement, de l'aménagement urbain et l'environnement, de l'alimentation et de l'agriculture et ceci tant au niveau national 
que régional et local (Buckett, 2010; Ståhl, Wismar, Ollila, Lahtinen et Kimmo Leppo, 2006). Aux États-Unis, la pratique des EIS est récente, des expériences d'EIS ont été entreprises par diverses organisations principalement dans l'état de Californie et particulièrement dans le domaine de l'éducation et de l'agriculture (Gagnon et Michaud, 2008). Le développement des EIS à travers le monde est diversifié aux niveaux national, régional et local. Une des conséquences est que le suivi des publications sur les EIS et sur les PPFS n'est pas toujours évident.

Plusieurs années après le début de leur mise en œuvre, il est possible d'étudier les résultats apportés par les EIS et les PPFS. Par exemple, une vaste étude portant sur l'efficacité de l'EIS menée auprès de 19 pays européens a conclu que cette pratique était capable d'influencer le processus de prise de décision et d'accroître la sensibilité des décideurs envers les déterminants de la santé (Wismar, Blau, Ernst et Figueras, 2007). De plus, une étude britannique indépendante a établi un rapport coût-bénéfice positif pour cette pratique (St-Pierre et Hamel, 2009; York Health Economics Consortium, 2006).

Dans le cas des administrations publiques ayant institutionnalisé les EIS ou les PPFS, on peut s'attendre à ce que des résultats soient finalement perceptibles après plus de dix ans de mise en œuvre. En effet, le Québec a mis en place en 2001 un article de loi appuyant la démarche de prise en compte de la santé dans les politiques publiques (Gouvernement du Québec, 2001, article 54). Nous proposons d'étudier les défis posés lors des différentes étapes de mise en œuvre de cette disposition législative et d'en dégager des pistes d'action pour les 10 années futures, à la lumière des 10 années passées, et des menaces actuelles ou à venir.

L'analyse de menaces est conseillée dans le champ de la santé publique pour informer de l'état actuel d'une situation et pour faire ressortir les axes d'action possibles à travers une matrice SWOT (Strengths - Weaknesses - Opportunities - Threats) ou AFOM (Atouts - Faiblesses - Opportunités - Menaces) (Van Wijngaarden, Scholten et van Wijk, 2012). L'étude que nous proposons consiste à relever les menaces, comprises dans le sens de l'analyse AFOM, et plus particulièrement celles qui sont en lien avec des aspects communicationnels. Se concentrer sur les menaces au sens de l'AFOM signifie regarder, dans l'environnement des PPFS, les facteurs défavorables aux PPFS, ceux qui peuvent ralentir, diminuer ou empêcher la mise en œuvre des PPFS. L'analyse des menaces semble donc utile pour améliorer la visibilité de l'action publique, en particulier pour informer l'action au regard des PPFS pour les années à venir.

L'article est divisé en trois sections.

La première section présente une description séquentielle des activités déployées depuis l'initiation de la démarche des PPFS au Québec. Nous résumons chacune des périodes d'implantation des PPFS pour en extraire les acteurs ${ }^{1}$ impliqués, l'engagement des organisations publiques envers les PPFS et la construction d'une appropriation commune de la prise en compte de la santé dans l'administration publique.

La seconde section permet d'analyser, pour le cas à l'étude, des menaces à la mise en œuvre des PPFS. Les menaces en date de 2013 correspondent aux barrières relevées par l'ensemble des acteurs œuvrant dans la mise en œuvre récente des PPFS, et qui peuvent

${ }^{1}$ Le terme acteur ici s'entend dans le sens large d'individus, groupes ou d'organisations. 
constituer une menace pour le futur des PPFS. Nous nous concentrons sur les menaces liées aux aspects communicationnels notamment entre les acteurs en charge de la politique transversale en question.

La troisième section porte sur une proposition de pistes d'action qui permettraient de réduire les menaces ou leurs effets afin de poursuivre, améliorer et pérenniser la mise en œuvre de la PPFS. Afin de déduire des pistes d'action crédibles, nous évoquons les alternatives provenant des expériences variées du Québec et de l'international qui répondraient aux menaces précédemment énoncées. Puis nous discutons de leur transférabilité au contexte des PPFS au Québec.

Les méthodes mises en œuvre pour chacune de ces trois sections, qui correspondent aux trois étapes de notre étude, sont présentées en annexe.

\section{Présentation de l'historique et des périodes d'implantation au Québec}

Bien que les périodes ne soient pas établies a priori et que leurs limites temporelles soient perméables, nous présentons ici trois périodes majeures dans la mise en œuvre des PPFS au Québec : période 1 (2001-2004) adoption de l'article 54, période 2 (2005-2008) mise en œuvre de l'article 54, période 3 amélioration continue (2009-2013) ${ }^{2}$. En parallèle, certaines stratégies indirectement liées aux PPFS se sont aussi déployées comme le Plan d'action gouvernemental de promotion des saines habitudes de vie et de prévention des problèmes reliés au poids, Investir pour l'avenir (Gouvernement du Québec, 2006).

\subsection{Période 1 : adoption de l'article 54 (2001-2004)}

Depuis l'introduction de l'article 54 de la Loi de Santé publique en 2001, les ministères ont l'obligation de consulter le Ministère de la Santé :

"Le ministre est d'office le conseiller du gouvernement sur toute question de santé publique. Il donne aux autres ministres tout avis qu'il estime opportun pour promouvoir la santé et adopter des politiques aptes à favoriser une amélioration de l'état de santé et du bien-être de la population. À ce titre, il doit être consulté lors de l'élaboration des mesures prévues par les lois et règlements qui pourraient avoir un impact significatif sur la santé de la population » (Gouvernement du Québec, 2001).

Cet article traduit la volonté du gouvernement de s'assurer de la prise en compte des effets potentiels des projets de lois et de règlements sur la santé et le bien-être de la population (St-Pierre et al., 2012). Le Ministère de la Santé a ainsi un pouvoir d'initiative lui permettant d'émettre des avis vers ses collègues dans tous les secteurs d'intervention du gouvernement, et ceci dans l'optique de favoriser le développement et l'adoption de PPFS (CCNPPS/INSPQ, 2012). L'article 54 est entré en vigueur en juin 2002, et représente un levier important pour l'élaboration et l'adoption de PPFS.

Peu de temps après son entrée en vigueur, une étude met en évidence une variation dans les modalités d'adoption de l'article 54 entre les ministères à vocation sociale, économique

\footnotetext{
${ }^{2}$ Nos sources de données ne nous permettent pas de couvrir certaines des étapes du cycle de développement d'une politique (Jones, 1970; Lasswell, 1956), notamment les phases d'émergence du problème, de mise à l'agenda et de terminaison.
} 
et culturelle, et sur l'acceptabilité politique de l'article 54 par les ministères (Auger, Bélanger et Grossemy, 2003; CCNPPS/INSPQ, 2012). Ainsi, il a été recommandé au Ministère de la Santé de favoriser une meilleure compréhension de la santé et de ses déterminants dans le but d'influencer les perceptions et de modifier les pratiques au sein, notamment, des ministères à vocation économique. Il est aussi suggéré que le Ministère de la Santé développe des actions interministérielles pouvant faciliter l'intégration de la santé dans les autres secteurs (Observatoire de l'administration publique, 2003).

Pendant la période 1, de 2001 à 2004, les acteurs impliqués sont essentiellement les professionnels et les gestionnaires de la fonction publique qui œuvrent au Ministère de la Santé et dans les ministères à vocation sociale.

\subsection{Période 2 : mise en œuvre de l'article 54 (2005-2008)}

La stratégie se déploie en deux volets, l'un administratif et l'autre scientifique. Le volet administratif couvre " la mise en œuvre d'un mécanisme intra gouvernemental d'EIS reposant sur un réseau de répondants ministériels; la production et la diffusion d'outils; une procédure interne de traitement des demandes d'avis des ministères promoteurs de projets de loi et de règlement. » (Gagnon et Michaud, 2008 ; Gagnon, Turgeon et Dallaire, 2008 ; Gagnon, Turgeon et Gagné, 2007).

Le volet scientifique couvre « le développement et le transfert des connaissances sur les politiques publiques favorables à la santé et au bien-être reposant sur la mise en place, le suivi et les retombées du programme de recherche sur les politiques publiques favorables à la santé et au bien-être ; l'entente entre le Ministère de la Santé et l'Institut national de santé publique du Québec ainsi qu'un programme de recherche universitaire »(Gagnon et Michaud, 2008; Gagnon et al., 2008 ; Gagnon et al., 2007).

Le Ministère de la Santé convie ainsi des partenaires scientifiques à participer à la mise en œuvre de la stratégie d'implantation de l'article 54. Des universitaires sont sollicités pour proposer des outils et suivre le déploiement de la stratégie. De plus, une entente écrite est passée entre le ministère et son institut de santé publique pour réaliser des projets ciblés, des recherches appliquées et du soutien au développement de projets ministériels.

Un bilan présente les activités menées et les ressources utilisées pour soutenir la recherche et le transfert des connaissances liés à l'application de l'article 54 entre 2002 à 2007 (CCNPPS/INSPQ, 2012; MSSS., 2008). En 2005, le Ministère de la Santé conduit un bilan des moyens utilisés, résultats obtenus et difficultés rencontrées (MSSS, 2005). Une recherche exploratoire et descriptive présente les actions concrètes privilégiées par les acteurs de santé publique et celles menées aux niveaux central et régional en matière de politiques favorables à la santé au Québec (Gagnon et al., 2007).

Les travaux de Bourgault et coll. (Bourgault, Dupuy et Turgeon, 2008) présentent une typologie et les conditions de succès d'une gestion horizontale en comparant plusieurs situations au Québec et au Canada, incluant le cas de la mise en œuvre de l'article 54. Les auteurs observent que l'adhésion à l'article 54 ne se faisait pas de manière spontanée, et que cela nécessite une approche proactive du Ministère de la Santé. D'autres auteurs présentent des cadres conceptuels et les mécanismes de mise en œuvre d'une gouvernance intégrée de multiples secteurs d'activités gouvernementales (Gagnon et Kouri, 2008) qui peuvent s'appliquer à l'article 54 . 
Pendant la période 2, de 2005 à 2008, les acteurs impliqués sont essentiellement les professionnels et les gestionnaires de la fonction publique qui œuvrent au Ministère de la Santé et plusieurs équipes de scientifiques fournissant des données probantes.

\subsection{Période 3 : Amélioration continue (2009-2013)}

Cette période est caractérisée par une volonté marquée d'amélioration continue et de changement. Un troisième axe a d'ailleurs été explicité dans la stratégie de mise en œuvre de l'article 54 :

«3) l'amélioration continue de la stratégie d'implantation avec notamment l'évaluation des pratiques de prise en compte de la santé » (Smits, Denis, Preval et Duranceau, 2013).

Pendant cette période, plusieurs actions ont été déployées pour impliquer le Québec dans des échanges interprovinciaux, internationaux, et dans des comparaisons internationales. Les liens avec les expériences hors frontières se sont réalisés à travers : une table ronde sur les EIS au Canada où les participants ont souligné l'importance de la complémentarité entre les niveaux central, local et régional (St-Pierre, 2008), un article comparatif de la stratégie de mise en œuvre des PPFS entre l'Australie et le Québec (St-Pierre et Gauvin, 2010), l'accueil de la conférence internationale sur les EIS en août 2012 (Smits et al., 2013). Par ailleurs, le Ministère de la Santé envisage de combiner des EIS et des actions visant les PPFS, avec les évaluations d'impacts intégrés au niveau ministériel (St-Pierre et Gauvin, 2010).

Une nouvelle étude apporte une mise à jour des résultats atteints dans plusieurs secteurs et des défis sectoriels et intersectoriels restants (Smits et al., 2013). Nous présentons ces défis en détail dans la section suivante.

Pendant la période 3, de 2009 à 2013, les acteurs impliqués sont essentiellement les professionnels et les gestionnaires de la fonction publique qui œuvrent au Ministère de la Santé, les professionnels et les gestionnaires dans les ministères sectoriels, plusieurs équipes de scientifiques du Québec et hors des frontières québécoises.

Après douze années d'implantation de l'article 54, les voies à explorer pour la prochaine période devront prendre en compte le contexte actuel, les changements administratifs en cours, et l'évolution de la sensibilisation de certains ministères à l'égard de la santé. Nous proposons, dans la prochaine section, d'analyser les menaces principales qui se présentent. Le but est de dégager des apprentissages pour les années à venir. Ces apprentissages sont tirés de la stratégie d'implantation des PPFS au Québec et plus largement d'initiatives appelant l'intervention et la coordination intersectorielle et interministérielle.

\section{Menaces liées à la prise en compte de la santé dans les politiques publiques}

La menace est ici comprise comme un élément dépeint par plusieurs acteurs interrogés et qui peut compromettre l'atteinte des objectifs, ici qui compromettrait la prise en compte de la santé dans les politiques publiques de l'administration publique du Québec. Les menaces peuvent être intrinsèques aux pratiques des organisations, ou provenir de l'environnement dans lequel évoluent les organisations.

Il n'est pas pertinent pour les besoins de l'article de procéder à une énumération de l'ensemble des menaces à la mise en œuvre de l'article 54 puis d'en apprécier systéma- 
tiquement leur probabilité et gravité. Nous débutons plutôt avec l'ensemble des menaces avérées ou raisonnablement probables qui ont été énumérées par au moins deux acteurs impliqués dans la mise en œuvre de la prise en compte de la santé dans les politiques publiques au Québec.

Parmi les menaces relevées, certaines sont relativement communes à tout projet, qu'il soit transversal ou non. Ainsi des contraintes financières (manque de ressources financières), ou légales (interdiction de certaines actions ou suppression de l'article 54) peuvent fragiliser les processus en place. Nous ne détaillerons pas ces deux menaces et préférons nous concentrer sur les autres menaces discutées. Ces menaces sont liées à aspects communicationnels.

\subsection{La cohérence de l'émetteur en cours de consolidation}

Le discours sur l'importance des PPFS et les actions afférentes sont menés par le Ministère de la Santé, plus précisément par une de ses directions (direction de santé publique) avec le concours des équipes scientifiques. Ce discours n'est pas toujours perçu comme une priorité par l'ensemble du ministère de la Santé. En effet, le discours des dirigeants peut être divergent de celui des scientifiques voire des professionnels de son administration. Ainsi, pour une réglementation donnée, "la santé des gens pouvait être mise en cause [mise à mal avec cette proposition de réforme]... en réponse à cela, le premier ministre [apparaît] à la télévision » (Question sur les barrières) pour faire la promotion de l'activité économique reliée à la réglementation plutôt que pour souligner ses effets sur la santé.

Le discours sur les PPFS n'est pas non plus toujours perçu comme une priorité par les autres ministères lorsque la structure administrative en charge de mettre en œuvre l'article 54 a un pouvoir limité. En effet, les ministères partenaires, agriculture, développement économique, etc., peuvent ne pas prêter la considération voulue aux PPFS dans la mesure où le Ministère de la Santé et sa direction générale ne contactent pas les autres directions générales des ministères directement mais qu'une des directions du Ministère de la Santé, la direction de santé publique, tente de contacter une autre direction générale. Certains voient mal comment l'article 54 «qui est à l'intérieur d'une direction d'un ministère va transcender l'ensemble des ministères » (Répondant, ministère. Question sur la visibilité de la santé publique). L'arrimage au sein du Ministère de la Santé entre les différentes directions administratives, ainsi qu'un arrimage entre ces directions et les directions concernées dans d'autres ministères pourraient être plus étroits.

\subsection{Un récepteur technique et populationnel plutôt que politico-administratif}

L'importance de prendre en compte la santé dans les politiques publiques est véhiculée en direction de deux publics cibles : la population générale qui peut lire les communiqués et études produites, et le personnel, les professionnels et gestionnaires des ministères, qui participent aux réunions avec le Ministère de la Santé. Or les décisions concernant les lois, règlements, projets majeurs des ministères sont aussi du ressort ultime des hauts fonctionnaires et des ministres. Au-delà de l'argumentation par les données probantes, ultimement «c'est un débat quasi... c'est plus politique, c'est un choix » (Répondant, ministère. Question sur la place accordée aux données probantes). Ces choix se font aussi sur une base émotionnelle et individuelle pour des décideurs. Un répondant raconte «le 
ministre est allé au-delà même de ce que les évidences de recherche [montraient], sur la base d'un cas personnel qui l'avait touché » (Répondant, ministère. Question sur la place accordée aux données probantes), il a pris une décision éloignée des données scientifiques.

La cible ou le récepteur du discours sur les PPFS est essentiellement technique à ce jour et orientée vers la population, plutôt qu'orienté vers la sphère politico-administrative.

\subsection{Un médium en définition}

Afin de faire parvenir le message à plusieurs audiences (population, sphère politique, sphère administrative), les moyens et stratégies déployées reposent entre autres sur des supports matériels comme des pamphlets, rapports, etc., et sur des rencontres entre ministères.

Quelques expériences fâcheuses pour les PPFS mettent en avant le pouvoir de certains médiums. Par exemple, nous venons de mentionner que dans une situation, un ministre a pris une décision sur une base émotionnelle allant à l'encontre des données probantes. Le cas personnel qui avait touché le ministre avait été «monté en épingle » par "les médias » qui « utilisent les anecdotes » (Répondant, ministère. Question sur la visibilité de la santé publique).

Dans une autre situation, l'annonce d'un plan a été affectée et bloquée puisque celuici aurait pu avoir des répercussions sur l'image qu'un ministre entend conserver : «Un ministre va être fier de son plan d'action, ce qui peut porter ombrage aux autres. Pourquoi le ministre responsable de x laisserait-il le ministre de z prendre le crédit de l'ensemble des mesures qui sont dans le plan d'action interministériel, alors que plusieurs autres mesures importantes [dépendant de z] y sont? » (Répondant, ministère. Question sur la visibilité de la santé publique). Une politique transversale peut ainsi être entravée, et a contrario facilitée, en prenant en considération l'importance de l'image publique des décideurs.

Finalement, alors qu'un discours sur les PPFS peut à lui seul ne pas être facilement entendu, il peut se greffer à d'autres actions pour lesquelles une audience est acquise. Ainsi l'implantation de la stratégie de développement durable dans chaque ministère a permis d'apporter aussi un volet santé dans les réflexions. À l'inverse, certains acteurs craignent, à long terme, que la stratégie de développement durable absorbe, et par l'occasion, dilue la voix du secteur de la santé publique parmi l'ensemble des préoccupations couvertes, santé, sociale, économique, environnement : «le volet santé dans les impacts environnementaux n'est pas complet là » (Répondant, ministère. Question sur la visibilité de la santé publique).

Ces trois situations illustrent des médiums, image politique, média, intégration dans des actions existantes, qui ont été employés à dessein ou non et qui ont des répercussions sur les PPFS. Les médias, l'image politique ainsi que l'intégration dans des actions existantes déjà porteuses apparaissent aussi comme des moyens pour véhiculer, ou bloquer, le message sur les PPFS.

Finalement, les menaces sont fortement ancrées dans le contexte de fonctionnement en silo des administrations, dans une visibilité limitée de la mise en œuvre de l'article 54, dans la qualité de l'arrimage interne de la direction de santé publique du ministère de la santé avec l'ensemble du ministère de la santé, et dans la place accordée aux données probantes dans la prise de décision. La mise en œuvre de l'article 54 requiert une cohérence intra et interministérielle sans laquelle le message perd de sa force. Les acteurs qu'il semble impératif de rejoindre regroupent à la fois les hauts décideurs de politiques publiques, les 
administrateurs et la population, ultime bénéficiaire des politiques publiques. La manière d'agir dans le futur s'apparente fortement à une stratégie multimodale pour permettre un tissage de liens et de collaborations partenariales efficaces avec d'autres ministères.

\section{Pistes d'action futures}

Les pistes sont comprises comme les réponses qui peuvent être raisonnablement envisagées dans un futur proche pour des praticiens face aux menaces énumérés précédemment. Les pistes doivent s'inscrire dans l'esprit de la politique publique étudiée, ici les EIS et les PPFS, et doivent/peuvent être documentées par des expériences antérieures.

Nous présentons séquentiellement les suggestions citées dans la littérature sur l'action transversale en lien avec la communication, puis une sélection d'actions possibles au Québec, et enfin une proposition et une traduction à travers des exemples concrets pour des gestionnaires.

Les pistes d'action sont : un arc à deux cordes (vers les décideurs, et vers la population); un leadership sur la gestion horizontale, et une publicisation des données probantes.

\subsection{Un arc à deux cordes}

Cette piste consiste à établir des liens, formels ou informels vers deux groupes ciblés par la mise en œuvre des EIS et des PPFS. Lorsque les démarches sont mises en œuvre dans les administrations publiques pour alimenter la prise de décision, des décideurs ultimes sont à rejoindre : ce groupe visé inclut des ministres et sous-ministres adjoints (Gow, 1995). Lorsque les démarches sont davantage orientées vers un processus participatif et de mobilisation de la voie citoyenne (Lehoux, Daudelin et Abelson, 2012 ; Loncle et Rouyer, 2004 ; Warin, 1999), des mesures et actions d'implication de citoyens ou de leurs représentants sont (à) envisagées (er) à différents stades du processus évaluatif.

Une des pistes pour le Ministère de la Santé et ses partenaires scientifiques est de rejoindre les hauts décideurs et les citoyens. Ce rôle permettrait de répondre à plusieurs menaces relevées précédemment. En effet, la visibilité des enjeux de santé publique auprès des citoyens et des décideurs, et la place que les décideurs accordent aux données produites dans leur décision ultime pourraient ainsi être renforcées.

Des auteurs mettent d'ailleurs en avant que l'institutionnalisation des EIS requiert l'engagement des responsables gouvernementaux, un leadership fort, et le partage d'informations sur les déterminants de la santé (McQueen, Wismar, Vivian, Jones et Maggie, 2012; Ståhl et al., 2006) et dans certains cas un cadre légal ou la promotion et le développement d'une culture EIS (Banken, 2001). Par ailleurs, les approches EIS se distinguent par la place accordée aux avis d'experts (Harris-Roxas, 2012) ou au renforcement de la voix citoyenne (CCNPPS/INSPQ, 2012; Harris-Roxas, 2012; St-Pierre et Hamel, 2009).

Une telle option, de communication vers les hauts décideurs et d'implication des citoyens apparaît réalisable dans le contexte actuel et dans un futur proche au sein de l'administration publique du Québec. Concernant les actions vers les décideurs, un cadre légal appuie les interventions du secteur de la santé au niveau politique. De plus, des liens formels et réguliers ont été établis avec certains ministères et pourraient être des exemples à dupliquer 
avec d'autres ministères. Concernant les liens vers les citoyens, des groupes de citoyens sont actuellement invités dans des discussions préliminaires avant le dépôt de certains dossiers ministériels pour vote à l'assemblée. Ces groupes pourraient être sensibilisés à la prise en compte de la santé dans les politiques publiques.

Nous partageons quelques idées concrètes d'action : échange d'anecdotes avec les hauts décideurs sur des politiques publiques peu favorables à la santé, affichage des anecdotes sur les lieux de passage dans les ministères (ex. campagne publicitaire dans les couloirs), plaidoyer ciblé des alternatives dans le cas d'une politique publique peu favorable à la santé, prix de la PPFS, prix de la PP peu favorable à la santé, veille internationale sur les thèmes en émergence dans des secteurs ciblés, encouragement d'invitations de professionnels de la santé lors de rencontres et déplacements de décideurs, partage d'anecdotes lors de rencontres informelles avec les élus et décideurs, etc.

\subsection{Un leadership concernant la gestion horizontale}

Cette option consiste à encourager une réflexion au sein de l'ensemble de l'administration publique sur le thème de la gestion horizontale/transversale et à enclencher une dynamique de gestion horizontale. En s'incluant au sein des instances décisionnelles concernant des projets horizontaux, les acteurs du secteur de la santé publique (professionnels, gestionnaires, scientifiques) s'assurent que soit visible la prise en compte de la santé sur des dossiers largement intersectoriels. Nonobstant leur participation à des projets ponctuels, par exemple sur le questionnement quant au gaz de schiste, une part proactive peut être prise dans les réflexions sur les modalités de gestion horizontale à l'échelle de l'administration publique dans sa globalité. Par exemple, quels sont les moyens appropriés pour piloter les projets interministériels ? Quels sont les outils, ressources, expertises nécessaires et utilisables pour l'ensemble des projets interministériels?

Ce rôle de l'administration publique dans les mécanismes de gestion horizontale permettrait de répondre à plusieurs des menaces relevées. Les menaces liées à la gestion en silo et à la multiplication des actions horizontales pourraient être ainsi prises en compte.

Des auteurs mettent d'ailleurs en avant la collaboration interorganisationnelle parmi les modalités de gestion intéressantes. Les décideurs politiques ont tendance à appliquer l'approche pangouvernementale, encore appelée gestion horizontale ou administration en réseau (Agranoff et McGuire, 2001 ; Bourgault et Lapierre, 2000). Les gouvernements ont consenti des efforts pour améliorer la gestion des affaires publiques, notamment via le décloisonnement de la gestion (Burns et Yeaton, 2008 ; Klijn, 2008; O’Toole, 2004; Ospina et Saz-Carranza, 2010). Une telle collaboration entre les acteurs des différents secteurs dépend du degré d'interaction et d'interdépendance entre eux et ne se base pas simplement sur le partage d'information (CRICH, 2011). Dans le secteur de la santé, une culture de coopération entre les différents secteurs et leurs leaders devrait permettre une meilleure compréhension des déterminants de la santé (Kickbusch et Buckett, 2010; Ståhl et al., 2006).

Une telle option de leadership concernant la gestion horizontale apparaît réalisable dans le contexte actuel et dans un futur proche au sein de l'administration publique du Québec. Plusieurs organisations peuvent initier, participer, et dynamiser une réflexion sur la gestion horizontale des politiques publiques, que ce soient des ministères sectoriels ou encore centraux. Le Ministère de la Santé a déjà mené quelques actions en 
ce sens. Il a récemment pris l'initiative de démarcher un ministère qui déployait un projet majeur en termes de développement économique et de santé. Une étude a aussi été récemment commandée par le secteur de la santé pour documenter les options empruntées par plusieurs pays pour intégrer la prise en compte de la santé parmi des procédures élargies d'évaluation comme les évaluations d'impact intégrées. Cet élan permet au secteur de la santé de se positionner parmi le groupe de réflexion sur certains enjeux de la gestion horizontale et d'occuper ainsi un espace stratégique dans les changements au sein de l'administration publique. Un tel élan pourrait être poursuivi pour assurer un positionnement du secteur de la santé à l'avant-garde de changements administratifs majeurs concernant la gestion horizontale.

Afin de tendre vers une réflexion sur les processus de gestion horizontale dans l'administration publique, quelques idées concrètes seraient par exemple de mettre en place des rencontres sur le bilan des actions horizontales des dernières années, d'organiser une session sur les leçons transférables entre les évaluations de la mise en œuvre des EIS et celle de la stratégie de développement durable, etc.

\subsection{Une publicisation des données probantes}

Cette option consiste à mettre sur pied une démarche de communication pour s'assurer que les données probantes sont transmises avec un message et un véhicule de communication adéquats pour les ministères. En s'assurant que les ministères ont l'information dont ils ont besoin, soit une information directement utilisable et traduite par rapport à leur contexte sectoriel, et qui s'appuie sur les sources d'information du secteur de la santé (données scientifiques, histoires de cas, expériences de professionnels, etc.), les acteurs du secteur de la santé publique (professionnels, gestionnaires, scientifiques) s'assurent d'un plus grand intérêt et d'une facilité d'intégration de la santé dans les dossiers sectoriels.

Ce rôle dans la publicisation des informations permettrait de répondre à plusieurs menaces relevées. Les menaces liées à la place des données probantes dans les décisions et à la visibilité du secteur de la santé dans les dossiers sectoriels pourraient être ainsi davantage prises en compte.

Des auteurs mettent d'ailleurs en avant que la gamme des guides EIS s'est considérablement élargie au cours de la dernière décennie (St-Pierre et Hamel, 2009), fournissant une gamme de données sur les interventions et leurs effets sur la santé. L'utilisation de guides et d'outils dérivés des EIS reste cependant limitée (Mendell, 2010). Les auteurs soulignent qu'il est nécessaire de prendre en considération le fait que les données probantes vont audelà des données scientifiques et doivent, pour être utilisées, être traduites afin de faire sens dans le contexte d'exercice de décideurs (Boyle, 2009), ou de praticiens.

Une telle option de publicisation des données probantes apparaît réalisable dans le contexte actuel et dans un futur proche au sein de l'administration publique du Québec. Concernant la traduction de données probantes, un exercice a eu lieu il y a quelques années pour co-développer, entre le Ministère de la Santé et un ministère sectoriel, un support de communication conjoint (Smits et al., 2013). Les acteurs de la santé ont ainsi dû s'approprier les enjeux d'un autre secteur, et le secteur partenaire a reçu une information traduite sur les impacts santé pour son secteur. Une publicité basée sur des données traduites semble plus favorable à une utilisation dans les secteurs de compétence des 
ministères concernés, et requerrait une traduction impliquant directement les acteurs du secteur de la santé.

En plus du contenu traduit, des actions de publicité concrètes et plus traditionnelles peuvent être envisagées : diffusion de documents traduits, exposition dans les secteurs de compétence des ministères concernés pour sensibiliser à la santé, stratégie de communication mobilisante et interactive avec les ministères, avec les regroupements communautaires locaux, inclusion d'exemples dans des cours ciblés ou des programmes d'enseignement, etc.

\section{Conclusions et discussions}

Nous avons présenté le cas de la mise en œuvre d'une politique publique qui concerne l'ensemble des ministères : la prise en compte de la santé dans les politiques publiques au Québec. Il s'agit de proposer une analyse d'une politique publique horizontale à travers l'étude des menaces à sa mise en œuvre et de sa pérennité. Après une brève présentation des phases d'implantation de la politique sur plus de dix ans, nous présentons les menaces tangibles encourues pour la poursuite de la mise en œuvre, ainsi qu'une analyse des pistes d'action envisageables pour répondre à ces menaces.

Les pistes envisageables dans l'environnement actuel et dans un futur proche au sein de l'administration publique du Québec permettraient de répondre en partie aux menaces relevées. Les pistes d'action font référence à : un arc à deux cordes (les décideurs et la population), un leadership sur la gestion horizontale, une publicisation des données probantes.

Ces pistes appellent un positionnement avec des partenaires à l'intérieur du Ministère de la Santé, des décideurs d'autres ministères, et une participation citoyenne active. Par là même, les pistes évoquées appellent les acteurs de la mise en œuvre de l'article 54 et certains partenaires à endosser un rôle inhabituel. Bien qu'inhabituel, ce rôle ne constitue pas un changement vers l'inconnu puisque des actions ponctuelles au cours des années antérieures ont été menées en ce sens avec, par exemple, des échanges entamés avec le ministère du développement durable et des parcs sur le développement d'une des régions économiquement prometteuse du Québec. Il s'agit plutôt d'augmenter la fréquence d'actions particulière, voire mettre ce rôle davantage au centre des énergies et du temps déployé. Dans cette perspective, la prochaine période d'implantation, période 4, correspondrait à une prise de risque dans les rôles à occuper et les interfaces à déployer.

Il est évidemment envisageable de poursuivre dans la lignée entamée en période 3 en renforçant les actions mises en œuvre sans modifier ou ajouter de changements majeurs, au risque de lire des résultats similaires et consolidés, et de mettre en évidence des barrières similaires dans quelques années. Nous abondons dans le sens de Ison pour qui l'amélioration des EIS ne doit pas perdre de vue la consolidation des acquis ${ }^{3}$ (Ison, 2012). Il est

\footnotetext{
3 "Although there is undoubtedly scope for the redesign or adaptation of HIA, it is important not to lose sight of the gains already made from introducing and implementing HIA highlighted in this and other evaluations, e.g. improving understanding of health (particularly the social model) and the determinants of health, putting health on the agenda of policy- and decision makers, initiating or improving intersectoral working on health, and influencing decision making about proposals." (Ison, 2012).
} 
aussi important de «s'assurer que les apprentissages soient réutilisés pour permettre une meilleure intégration de la prise en compte de la santé dans les politiques et les décisions en général ${ }^{4}$. » (Ison, 2012).

La limite de notre analyse repose sur l'exhaustivité des menaces relevées. En effet, les menaces que nous avons considérées sont les menaces actuelles et perçues par les acteurs. Les menaces émergentes dans un futur proche n'ont pas été étudiées. Une analyse intégrant un niveau de menace moins probable pourrait être conduite. Toutefois, l'intégration de nouvelles menaces ne devrait pas invalider les pistes d'action discutées mais ajouter de nouvelles pistes, à moins de bouleversements rapides et majeurs dans l'administration publique.

Dans quelle mesure la démarche que nous avons empruntée (l'identification de menaces liées à des aspects communicationnels, la suggestion d'actions à apporter inspirée de la littérature, et l'analyse de la faisabilité dans le contexte du Québec) serait davantage pertinente pour des initiatives publiques similaires aux EIS et aux PPFS en comparaison à une évaluation? Une évaluation peut porter sur une gamme étendue de dimensions telles que les ressources, les activités, et les résultats pour établir des constats principalement sur les actions passées. Les facteurs facilitateurs et barrières peuvent aussi être relevés. Pour cet article, l'analyse a consisté à identifier uniquement les menaces et les pistes pour y faire face. En identifiant les menaces par entrevues, il apparaît que des pistes d'action peuvent assez facilement et rapidement être identifiées pour orienter la mise en œuvre future. Il reste à étudier si une évaluation qui porterait sur un ensemble de dimensions mènerait à des conclusions et des orientations similaires à celles que nous avons mises en évidence ici. La démarche employée nous paraît être une alternative pour documenter une situation de façon systématique et ordonnancée et pour en déduire logiquement des axes d'intervention.

Dans quelle mesure les menaces et pistes d'action que nous avons identifiées sont-elles généralisables pour des initiatives publiques similaires aux EIS et au PPFS ? Des initiatives intersectorielles et interministérielles, comme une stratégie de développement durable, une stratégie de lutte contre la pauvreté, et une stratégie de lutte contre le changement climatique tirent certaines conclusions qui sont similaires à celles que nous avons notées après une dizaine d'années d'implantation des PPFS. À titre illustratif, dans la lutte contre les changements climatiques, le niveau d'intensité du leadership des hauts décideurs est aussi déplorée (David Suzuki Foundation, 2005, 2012). La production de données probantes (Bureau du Vérificateur général du Canada, 2012; Vérificateur Général, 2013) et leur diffusion font aussi partie des efforts soulignés dans plusieurs actions interministérielles au Québec (Bureau du Vérificateur général du Canada, 2012; Gouvernement du Québec, 2010). La participation citoyenne est aussi mise à l'honneur pour orienter de futurs plans d'action interministérielle (Gouvernement du Québec, 2010). Le travail hors des silos ministériels est explicitement mentionné parmi les obstacles posés par l'action interministérielle (Bureau du Vérificateur général du Canada, 2012; Gouvernement du Québec, 2010), etc. Comme certaines leçons se répètent d'une initiative horizontale à l'autre, des apprentissages croisés sur la mise en œuvre des actions horizontales pourraient être bénéfiques.

Ceci est bien entendu conditionné par les capacités d'apprentissage et de changement des administrations publiques. Bien que les apprentissages ne se mesurent pas nécessairement

\footnotetext{
${ }^{4}$ Traduit de : "to ensure that the learning points from HIA are taken forward in the drive to integrate a concern for health in policy and decision making."
} 
de la même façon dans le secteur public et privé, il est néanmoins possible de mesurer et de mettre en évidence des apprentissages organisationnels dans le secteur public (Rist, 1993). Échanger sur les leçons apprises d'une initiative transversale à l'autre semblerait fertile pour générer des apprentissages dans les organisations publiques concernées, et aller au-delà de la gestion d'initiatives transversales limitée dans le temps. Par ailleurs, mettre l'accent sur les modalités de communication entre les organisations publiques préoccupées par une même problématique transversale peut constituer un axe qu'il conviendrait d'explorer plus avant. Nous avons présenté les défis de communication entre les acteurs impliqués dans les PPFS. Certains auteurs proposent aussi de regarder davantage les épisodes de communication entre celles-ci (Blaschke, Schoeneborn et Seidl, 2012; Koschmann, Kuhn et Pfarrer, 2012; Werner, 1988). La perspective des « organisations fondées sur la communication » est relativement récente parmi les études sur les organisations (Blaschke et al., 2012 ; Boden, 1994; Putman et Nicotera, 2009). Elle emprunte à la littérature sur les réseaux et sur la communication. Cette perspective, plutôt que de considérer une organisation ou un réseau sous l'angle de ses structures ou de ses pratiques, conçoit une organisation comme une multiplicité d'épisodes de communication plus ou moins imbriqués. Les épisodes de communication, ces séquences où la communication se réalise de façon plus ou moins planifiée autour d'un thème, pourraient être utiles pour structurer les interactions entre les secteurs et les ministères au-delà de la transmission de multiples messages d'un ministère à l'autre. S'organiser autour des épisodes de communication pourrait permettre une meilleure cohérence et une continuité dans les échanges.

\section{Remerciements}

Le rapport initial a été subventionné par la Direction Générale de la Santé Publique du Ministère de la Santé et des Services Sociaux du Québec avec la collaboration de Lyne Jobin, et Caroline Druet. L'article a été rédigé entièrement par les co-auteurs. Il n'a été ni relu ni commenté par le Ministère de la Santé et des Services Sociaux. 


\section{Bibliographie}

Agranoff R. and McGuire M., 2001. Big Questions in Network Management Research. Journal of Public Administration Research and Theory 11, 295-326.

Auger J., Bélanger P. et Grossemy D., 2003. Dentification des enjeux sociétaux et sectoriels pour l'opérationnalisation du programme de transfert de connaissances et de recherche sur les politiques publiques favorables à la santé et au bien-être.

Banken R., 2001. Strategies for Institutionalizing HIA. World Health Organization, Regional Office for Europe, Copenhagen, European Centre for Health Policy ECHP, Brussels.

Blaschke S., Schoeneborn D. and Seidl D., 2012. Organizations as Networks of Communication Episodes: Turning the Network Perspective Inside Out. Organization Studies, 33(7), 879-906.

Boden D., 1994. The business of talk. Polity Press, Cambridge.

Bourgault J., Dupuy S. \& Turgeon J., 2008. Les conditions de succès des dispositifs interministériels. Groupe d'Études sur les Politiques Publiques et la Santé (GÉPPS).

Bourgault J. et Lapierre R., 2000. Horizontalité et gestion publique. Rapport final au Centre canadien de gestion, Réseau du Leadership, conseil régional fédéral - Conseil des hauts fonctionnaires du Québec et École nationale d'administration publique. Ottawa Centre canadien de gestion.

Boyle R., 2009. Evidence and Politicians : Can the two co-exist? In O. Rieper, Leeuw F.L., Ling T., Ed., The Evidence Book, Transaction Publishers, 163.

Buckett K., 2010. Health in all Policies : European Union Experiences and Perspectives. Health in all Policies. Adelaide 2010 Internationaal meeting. Public Health Bulletin SA, 7 (2).

Bureau du Vérificateur général du Canada, 2012. Rapport du commissaire à l'environnement et au développement durable. Chapitre 2. Ottawa Canada.

Burns T. J. and Yeaton K. G., 2008. Success Factors for Implementing Shared Services in Government. IBM Center for The Business of Government, Competition, Choice, and incentives Series. IBM.

CCNPPS/INSPQ, 2012. L'application de l'article 54 de la Loi sur la santé publique du Québec. Août 2012. Version préliminaire - pour discussion. Note documentaire. Centre de collaboration nationale sur les politiques publiques et la santé (CCNPPS). Institut national de santé publique du Québec (INSPQ).

CRICH, 2011. Getting Started with Health in All Policies: A Resource Pack. Health in All Policies: A Snapshot for Ontario - Results of a Realist-Informed Scoping Review of the Literature. Report to the Ministry of Health and Long-Term Care (Ontario). Centre for Research on Inner City Health (CRICH) in the Keenan Research Centre of the Li Ka Shing Knowledge Institute of St. Michael's Hospital.

CRICH, 2011. Getting Started with Health in All Policies: A Resource Pack. Health in All Policies: A Snapshot for Ontario - Results of a Realist-Informed Scoping Review of the Literature. Report to the Ministry of Health and Long-Term Care (Ontario). Centre for Research on Inner City Health (CRICH) in the Keenan Research Centre of the Li Ka Shing Knowledge Institute of St. Michael's Hospital.

David Suzuki Foundation, 2005. Un bilan disparate. Vancouver Canada.

David Suzuki Foundation, 2012. Un bilan disparate. Vancouver Canada.

Gagnon F. et Kouri D., 2008. Gouvernance intégrée et politiques publiques favorables à la santé : deux exemples canadiens. Rapport. Centre de collaboration nationale sur les politiques publiques et la santé Institut national de santé publique du Québec.

Gagnon F. et Michaud M., 2008. L'Évaluation d'impact sur la santé et la formulation de politiques publiques In S. Tremblay et V. Turcotte (Ed.), Série Transfert de connaissance, GEPPS.

Gagnon, F., Turgeon, J., \& Dallaire, C., 2008. L'évaluation d'impact sur la santé au Québec : lorsque la loi devient levier d'action. Revue d'analyse comparée en administration publique, 14 (2)

Gagnon F., Turgeon J. et Gagné, D., 2007. Les actions de la santé publique en matière de politiques favorables à la santé au Québec, de 1995 à 2005 - Perspectives centrales et régionales. Rapport final. GEPPS.

Loi de Santé Publique Article 54, 2001. 
Gouvernement du Québec, 2006. Plan d'Action gouvernemental de Promotion des saines habitudes de vie et de prévention des problèmes reliés au poids 2006-2012. Quebec, Canada.

Gouvernement du Québec, 2010. Plan d'action gouvernemental en matière de lutte contre la pauvreté et l'exclusion sociale 2004-2009 bilan de la cinquième année février 2010. Quebec, Canada.

Gow J., 1995. Propos dissidents sur l'imputabilité parlementaire des hauts fonctionnaires québécois. Administration Publique du Canada/Canadian public administration 38 (1), 145-149.

Harris-Roxas B., 2012. Health Impact Assessment. An introductory series of slides for the Maules Creek/Gunnedah health impact assessment meeting. Retrieved aout 2013, from http://fr.slideshare.net/benharrisroxas/healthimpactassessment

Ison E., 2012. Health Impact Assessment in a Network of European Cities. Journal of Urban Health.

Jones C. O., 1970. An Introduction to the Study of Public Policy. Wadsworth Publishing Company.

Kickbusch I. and Buckett K., 2010. Implementing Health in All Policies. Adelaide 2010.

Klijn E.-H., 2008. Complexity Theory and Public Administration: What's New? Public Management Review $10(3), 299-317$.

Knoepfel P., Larrue C. et Varone F., 2006. Analyse et pilotage des politiques publiques. V. Rüegger Ed., Zürich.

Koschmann M. A., Kuhn T. R. and Pfarrer M. D., 2012. A Communicative Framework of Value in Cross-Sector Partnerships. Academy of Management Review 37 (3), 332-354.

Lasswell H. D., 1956. The Decision Process: Seven Categories of Functional Analysis. University of Maryland, College of Business and Public Administration.

Lehoux P., Daudelin G. and Abelson J., 2012. The Unbearable Lightness of Citizens within Public Deliberation Processes. Social Science \& Medicine 74 (12), 1843-1850.

Loncle P. et Rouyer A., 2004. La participation des usagers : un enjeu de l'action publique locale. Revue française des affaires sociales 4, 133-154.

McQueen D. V., Wismar M., Vivian L., Jones C. M. and Maggie D., 2012. Intersectoral Governance for Health in All Policies. Série des études de l'Observatoire 26 (2).

Mendell A., 2010. Quatre types d'évaluation d'impact utilisés au Canada. Centre de collaboration nationale sur les politiques publiques et la santé (CCNPPS).

Miles M. and Huberman M., 1994. Qualitative data analysis (2 ed.). Sage Publication, London.

MSSS, 2005. Article 54 de la loi sur la santé publique. Bilan de mise en æuvre (document synthèse. Juin 2002 janvier 2005. santé et des services sociaux. Mai 2005.

MSSS, 2008. Bilan et perspectives 2002-2007. À la frontière des responsabilités des ministères et organismes publics : l'application de l'article 54 de la Loi sur la santé publique Quebec.

O’Toole L. J., 2004. The Theory-Practice Issue in Policy Implementation Research. Public Administration 82 (2), 309-329.

Observatoire de l'administration publique., 2003. Identification des enjeux sociétaux et sectoriels pour l'opérationnalisation du programme de transfert de connaissances et de recherche sur les politiques publiques favorables à la santé et au bien-être. ENAP.

Ospina S. M. and Saz-Carranza A., 2010. Paradox and Collaboration in Network Management. Administration \& Society 42 (4), 404-440.

Paillé P. et Mucchielli A., 2001. L'analyse qualitative en sciences humaines et sociales. Armand Colin, Paris.

Putman L. and Nicotera A., 2009. Building theories of organization : the constitutive role of communication. Routledge, NY.

Rist R. C., 1993. The Preconditions for Learning: Lessons from the Public Sector In F. Leeuw, Rist RC., Sonnichsen, RC., Ed., Can governments learn? Transactions Publishers, Piscataway.

Smits P., Denis J.-L., Preval J. et Duranceau M.-F., 2013. Évaluation des pratiques de prise en compte de la santé dans les politiques publiques. Rapport. ENAP, Québec. 
St-Pierre L., 2008. Table ronde canadienne portant sur l'évaluation d'impact sur la santé (ÉIS). Montréal, Québec, 22 février 2008. Centre de collaboration nationale sur les politiques publiques et la santé (CCNPPS).

St-Pierre L. and Gauvin F.-P., 2010. Intersectorial Governance for Health in All Policies: an integrated frame work. Public Health Bulletin SA, Health in All Policies, Adelaide 2010 International Meeting, 7 (2).

St-Pierre L. et Hamel G., 2009. Qu'est-ce que l'évaluation d'impact sur la santé (ÉIS) ? Fiche d'information pour des connaissances en matière de politiques publiques favorables à la santé. Centre de collaboration nationale sur les politiques publiques et la santé.

St-Pierre L., Viens C. et Tremblay, É., 2012. La pratique de l'évaluation d'impact sur la santé en Montérégie. Protocole d'évaluation In Centre de collaboration nationale sur les politiques publiques et la santé (CCNPPS), Agence de la santé et des services sociaux Direction de santé publique (Ed).

Ståhl T., Wismar M., Ollila E., Lahtinen E. and Kimmo Leppo, K., 2006. Health in All Policies. Prospects and potentials. "Europe for Health and Wealth". Finland.

Valentini H. et Saint-Pierre L., 2012. L'évaluation d'impact de la santé, une démarche structurée pour instaurer de la santé dans toutes les politiques. Santé Publique 6 (24), 479-482.

Van Wijngaarden J. D. H., Scholten G. R. M., van Wijk K. P., 2012. Strategic Analysis for Health Care Organizations: the Suitability of the SWOT-analysis. The International Journal of Health Planning and Management 27 (1), 34-49.

Vérificateur Général, 2013. Rapport du Vérificateur général du Québec à l'Assemblée nationale pour l'année 2012-2013 Rapport du commissaire au développement durable faits saillants. Québec.

Warin P., 1999. Les « ressortissants » dans les analyses des politiques publiques. Revue française de science politique 49 (1), 103-121.

Werner E., 1988. Toward a Theory of Communication and Cooperation for Multiagent Planning. Paper presented at the TARK Proceedings of the 2nd conference on Theoretical aspects of reasoning about knowledge.

WHO, 1999. Health Impact Assessment: Main Concepts and Suggested Approach: The Gothenburg Consensus Paper. WHO Regional Office for Europe Retrieved from http://www.who.dk/document/PAE/ Gothenburgpaper.pdf.

Wismar M., Blau J., Ernst K. and Figueras J., 2007. The Effectiveness of Health Impact Assessment-Scope and Limitations of Supporting Decision-Making in Europe. Copenhagen: World Health Organisation.

York Health Economics Consortium, 2006. Cost Benefit Analysis of Health Impact Assessment. Dept of Health, London. 


\section{Annexe}

\section{Méthodes}

La méthodologie employée se décline en trois phases. La phase 1 vise à comprendre les changements dans la mise en œuvre de la démarche des PPFS au Québec depuis plus d'une décennie. La phase 2 vise à identifier les menaces qui peuvent entraver le futur des PPFS. La phase 3 vise à proposer des pistes d'action.

Phase 1. Deux sources de données sont mobilisées : de la documentation comme source principale, et des entrevues concernant les PPFS au Québec. Les entrevues permettent de mettre en contexte les phases d'évolution. Pour l'analyse documentaire, nous avons d'abord ciblé les documents pouvant contenir des résultats et des recommandations sur des PPFS au Québec soit : les rapports d'évaluation et bilans d'activités réalisés dans le cadre de l'article de loi et des PPFS au Québec. Les documents ont permis de retracer une chronologie des événements et de dégager des phases d'évolution de la stratégie d'implantation des PPFS. Le passage d'une période à l'autre repose sur des rapprochements avec de nouveaux acteurs.

Pour les 61 entrevues, les personnes impliquées dans la mise en œuvre de PPFS au Québec ont été interrogées en 2012. Les entrevues portaient sur la place de la santé dans les PPFS, les activités déployées, les résultats obtenus, le processus décisionnel, le contexte. La sélection des personnes combine à la fois une méthode raisonnée avec des acteurs en charge de la mise en œuvre de PPFS (l'équipe concernée du Ministère de la Santé, les partenaires scientifiques, le réseau de répondants, certains fonctionnaires qui siègent sur des structures interministérielles), puis un échantillonnage boule de neige auprès de professionnels ou gestionnaires ciblés par le réseau de répondants.

Phase 2. Les mêmes entrevues ont permis d'identifier les menaces. La section de la grille d'entrevue qui portait sur le contexte couvrait des questions sur les facteurs favorables aux PPFS, les facteurs défavorables, les améliorations possibles, les supports existants.

Sur les personnes interrogées, une répartition a été assurée entre le Ministère de la Santé et les autres ministères, entre les ministères et le milieu académique (universités et institut de recherche), et entre les acteurs agissant dans le moment sur des PPFS spécifiques (exemple politique bio alimentaire, Agenda 21, etc.) et d'autres acteurs avec une expérience ou une connaissance antérieure des PPFS (Tableau 1. Répartition des entrevues).

Tableau 1. Répartition des entrevues

\begin{tabular}{|l|l|c|}
\hline \multicolumn{2}{|l|}{ Type } & Volume \\
\hline \multirow{4}{*}{ Appartenance institutionnelle } & Ministère de la Santé & 17 \\
\cline { 2 - 3 } & Autres ministères & 36 \\
\cline { 2 - 3 } & Partenaires scientifiques & 8 \\
\hline \multirow{2}{*}{ Acteur dans une PPFS spécifique } & Oui & 21 \\
\cline { 2 - 3 } & Non & 40 \\
\hline \multirow{4}{*}{ Niveau } & Bénéficiaire & 0 \\
\cline { 2 - 3 } & Politique & 61 \\
\cline { 2 - 3 } & Administratif & \\
\hline
\end{tabular}


L'analyse des entrevues repose sur une analyse par catégorisation des contenus (Miles \& Huberman, 1994 ; Paillé \& Mucchielli, 2001). Les entrevues ont été menées initialement par deux intervieweurs, puis par l'un ou l'autre d'entre eux. La grille de codage des entrevues est basée sur les thématiques abordées dans l'entrevue et sur les thèmes émergents lors des entrevues. Trois codeurs, dont les intervieweurs, ont procédé au codage. Lorsqu'un consensus a été atteint sur la grille, les définitions et une similarité du codage des sections de trois entrevues, l'ensemble des transcriptions a été divisé et codé sur le logiciel QDA Miner, version 4.0. L'analyse préliminaire des entrevues repose sur la rédaction de fiches descriptives, avec un résumé et des extraits de verbatim. Nous présentons dans cet article uniquement les résultats relatifs à la section contexte des entrevues et aux réponses sur les aspects communicationnels. En effet, les résultats sont de plusieurs ordres : ressources supplémentaires (manque de ressources financières), ressources légales (interdiction de certaines actions ou suppression de l'article 54), aspects communicationnels. À la lecture des résultats concernant le contexte, plusieurs éléments faisaient référence à des aspects communicationnels. Nous reprenons ces derniers éléments qui paraissent enrichissants dans le cas de politiques transversales. En effet, les dynamiques transversales dans les administrations publiques requièrent des collaborations inter organisationnelles avec, selon les propos des intervenants, de nécessaires échanges entre des secteurs habitués à des terminologies, des enjeux, et des pressions différentes, ne serait-ce qu'entre ministères à vocation économique et sociale. La compréhension commune d'une intervention publique interministérielle tire a priori bénéfice d'une communication efficace. De plus, les PPFS prennent appui sur une logique d'échange entre les secteurs qui sont chacun responsable de certains déterminants de la santé. Il est donc attendu que les ministères communiquent et s'ajustent pour soutenir des PPFS, ou tout du moins des politiques publiques « moins » défavorables à la santé que ce qui était initialement envisagé.

Nous avons privilégié l'organisation des menaces avec un cadre simple plutôt qu'une présentation sous forme de liste non structurée. Ainsi le cadre pour rassembler les menaces et les exposer dans cet article comprend quelques dimensions : émetteur, récepteur, message, médium. Ces dimensions sont issues de notre propre analogie avec les technologies de la communication. Les concepts du messager émetteur d'un message à travers un médium pour rejoindre un récepteur pourraient se décliner dans le cadre d'une politique publique et de la mise en œuvre de l'article 54 en : un messager qui est une autorité publique, ici le Ministère de la Santé, qui véhicule et s'assure de transmettre un message, ici la prise en compte de la santé dans les politiques publiques. Le récepteur, ou le public visé par les PPFS, est ultimement la population québécoise, ou le tiers gagnant (Knoepfel, Larrue, \& Varone, 2006; Warin, 1999). Dans le cadre de la politique transversale, les autres autorités administratives, les ministères, agissant sur les déterminants de la santé sont aussi des récepteurs visés. Le medium, ou moyen pour véhiculer le message, comporte entre autres des rencontres avec des personnes dans chaque ministère qui relaient le discours sur les PPFS (le réseau de répondants), et des supports de diffusion sur les déterminants de la santé.

Les entrevues, plus précisément la section contexte des entrevues et les réponses liées aux aspects communicationnels, ont permis de relever les menaces qui peuvent entraver le futur des PPFS et qui sont liées à l'émetteur, au récepteur, au message et au medium.

Phase 3. Les pistes d'action cohérentes avec les menaces liées à la communication ont été identifiées par étapes, et en se basant sur les données scientifiques et les expériences dans d'autres contextes. La sélection des pistes repose d'abord sur une identification des 
suggestions citées dans la littérature sur l'action transversale en lien avec la communication, puis une sélection d'actions possibles au Québec, et enfin une proposition et une traduction en des exemples concrets pour des gestionnaires.

$\mathrm{Au}$ moins trois pistes/options sont envisagées. Certaines pistes apportent une réponse à des menaces précédemment énumérées (les options futures), elles sont cohérentes avec les expériences d'autres juridictions (les options éprouvées), et présentent une probabilité d'être applicables dans le contexte du cas étudié, le Québec (les options applicables). 